\title{
Aditivos para el hormigón. Calidad y Normativa
}

PROF. DR. DEMETRIO GASPAR-TÉBAR ${ }^{*}$

ICCET/CSIC

MADRID - ESPAÑA

\section{$R E S U M E N$}

Los aditivos han perdido, en gran parte, su carácter de productos misteriosos con los que se consiguen transformaciones espectaculares en los hormigones, de tal modo que debidamente utilizados han contribuido eficazmente a su desarrollo, por lo que han pasado a ser un componente más del hormigón hasta tal punto que, en muchos casos, es necesaria su utilización.

En este artículo se da cuenta de la situación actual de la normativa española elaborada por el SC-2 (Aditivos) del CTN-83 (Hormigón) del AENOR y, de un modo especial, de la normativa europea realizada por el SC-3 (Admixtures for concrete)/TC 104 (Concrete) del CEN, tanto en lo que se refiere a los métodos de ensayo, como a las caracteristicas que se le exigen, especificaciones y criterios de conformidad aplicados a las principales familias de aditivos más utilizadas en la fabricación del hormigón, teniendo en cuenta que los aditivos se deben utilizar una vez que se conozcan sus efectos sobre los hormigones (especialmente, sobre hormigones y morteros de referencia) y que, además, se garantice que sus características se mantienen a lo largo del tiempo, estando avaladas por un control de calidad adecuado.

\section{$S U M M A R Y$}

The admixtures have lost a large part of their character of mysterious propducts with which spectacular transformations of concretes are achieved. On the other hand, they fave effectively contributed (when used properly) to the development of concretes and have become anhother one of their components to such an extent that, in many cases, their use is necessary.

This paper deals with the current situation of the Spanish Standard and, specially, of the European Standard elaborated by the SC-3 (Admixtures for concrete)/TC 104 (Concrete) of the CEN, where it analysis the testing methods and the characteristics required, specifications and conformity criteria to the principal families of admixtures most used in the concrete manufacturing, taking into account the fact that the admixtures must used once their effects on the concrete are know and that the maintaining of their characteristics must be quaranteed by an adequate quality control.

\section{1.- INTRODUCCIÓN}

El incremento que ha experimentado el empleo del hormigón, así como la aparición de nuevas técnicas de preparación del mismo y de puesta en obra, están intimamente ligados al uso de productos adicionados al hormigón en pequeña cantidad (menor del $5 \%$ en masa del contenido de cemento en el hormigón), con lo que se consigue mejorar alguna o algunas de sus propiedades; estos productos se conocen con el nombre de aditivos.
La posibilidad de conseguir con los hormigones tradicionales materiales capaces de satisfacer:

* las exigencias que, en general, han impuesto los avances tecnológicos,

* la estabilidad frente a unas determinadas condiciones ambientales,

* aumentar el rendimiento en la elaboración, transporte y puesta en obra del hormigón

\footnotetext{
*Presidente del SC-2 "Aditivos" del CTN-83 "Hormigón" del AENOR.
} 


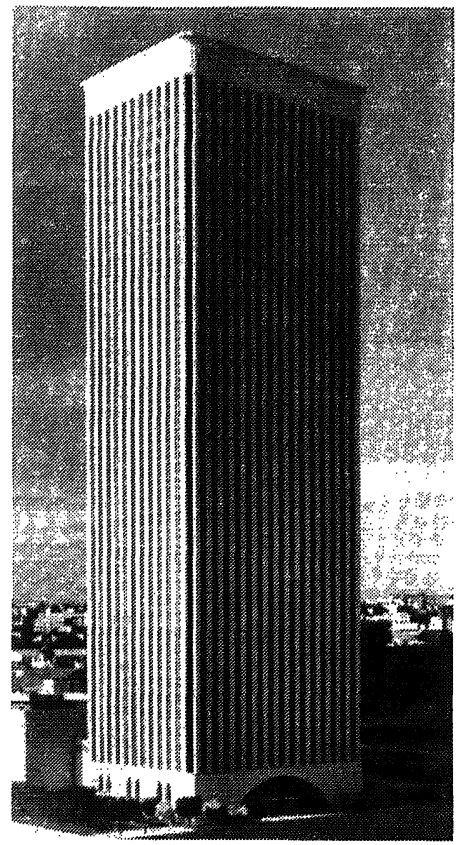

Torre Picasso, Madrid

es limitada, no permitiento encontrar, en la mayoría de los casos, una formulación tal que satisfaga las mencionadas exigencias para obtener productos más competitivos en los aspectos técnicos y económicos frente a otros materiales.

Además, se debe considerar que la industria del cemento ha experimentado, en los últimos años, una evolución exponencial no sólo en los conocimientos físico-químicos del cemento, y de un modo especial del clínker, sino en los procesos de producción y en la tecnología correspondiente, sin olvidar la existencia de cementos sin y con adiciones (diversas en cantidades variables), que figuran en la norma española UNE 80-301.

Por otra parte, se debe tener en cuenta que la industria química moderna ha puesto a disposición de la industria del hormigón una serie amplia de productos de síntesis y de subproductos que han tenido una influencia muy notable en su desarrollo, al permitir que las características o propiedades del hormigón se modifiquen en el sentido deseado.

El problema que plantea el conocimiento de la acción de los aditivos aplicados al hormigón es sin duda complejo, ya que se ha de tener un conocimiento profundo de la físico-química de los cementos y de los diferentes productos o sustancias que componen dichos aditivos, del mecanismo de los procesos que tienen lugar entre ambos (cemento-aditivo) durante el proceso de hidratación, así como sobre su influencia en laspropiedades del hormigón, por lo que dado el número y complejidad de las variables que intevienen se precisa una colaboración amplia entre técnicos de distintas especialidades.

Todo ello ha motivado la necesidad de realizar estudios profundos -en los que los aditivos han desempeñado un papel fundamental- para conocer la acción de dichos productos sobre el sistema complejo cemento-agua, sin descartar a los áridos y a las armaduras.

La importancia que ha adquirido el empleo de los aditivos en la elaboración del hormigón ha hecho que, actualmente, la normativa nacional e internacional, así como los Pliegos - Instrucciones -Códigos - etc., consideren a estos productos como uno de los componentes del hormigón. Por ejemplo, en la prenorma ENV 206:90, punto 3.1, del apartado 3, titulada "Concrete - performance, production, placing and compliance criteria" se define al hormigón como:

"... un material formado por una mezcla de cemento, de áridos finos y gruesos, y de agua, y producido por endurecimiento de la pasta de cemento (cemento $y$ agua), además de estos compuestos básicos, puede tener -también- aditivos y/o adiciones".

Del mismo modo, en los prEN 998-1:1993 y prEN 9982:1993, se definen a los morteros de revoco/enlucido, en el primero, y de albañilería, en el segundo, como:

"Mezcla de uno o varios conglomerantes inorgánicos, de áridos, de agua y a veces de aditivos $y / o$ adiciones".

La instrucción para el Proyecto y la Ejecución de Obras de Hormigón en masa 0 armado en el Artículo $8^{\circ}$ (otros componentes del hormigón) señala:

"También pueden utilizarse como componentes del hormigón los aditivos y adiciones, siempre que se justifique mediante los oportunos ensayos, que la sustancia agregada en las proporciones y condiciones previstas produce el efecto deseado sin perturbar excesivamente las restantes caracteristicas del hormigón ni representar peligro para la durabilidad del hormigón ni para la corrosión de las armaduras".

\section{2.- EMPLEO DE ADITIVOS}

La gran variedad de aditivos y, aun más, la enorme cantidad de productos inorgánicos y orgánicos que intervienen en su composición, se debe a que las distintas características y propiedades del hormigón y de los morteros se pueden ver influenciadas por numerosos compuestos, produciendo modificaciones en:

* la reología; aumentando la trabajabilidad para una 


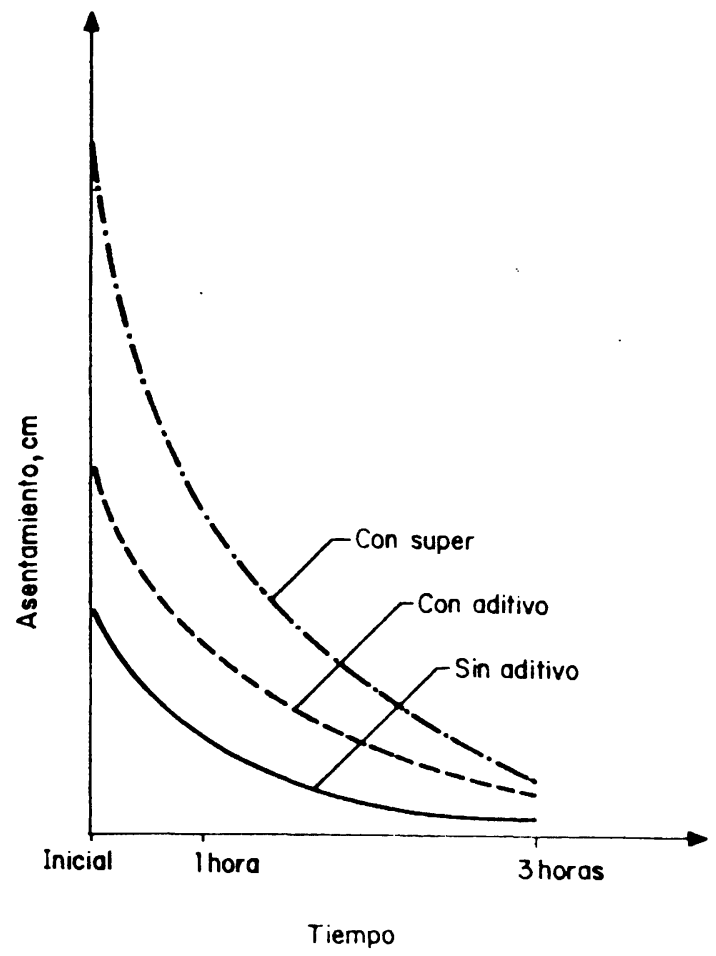

Fig. 1.- Trabajabilidad del hormigón: influencia del tipo de aditivo. (Guide to chemical admixtures for concrete. Concrete Society). Technical Report $n^{\circ}$ 18. London, 1980).
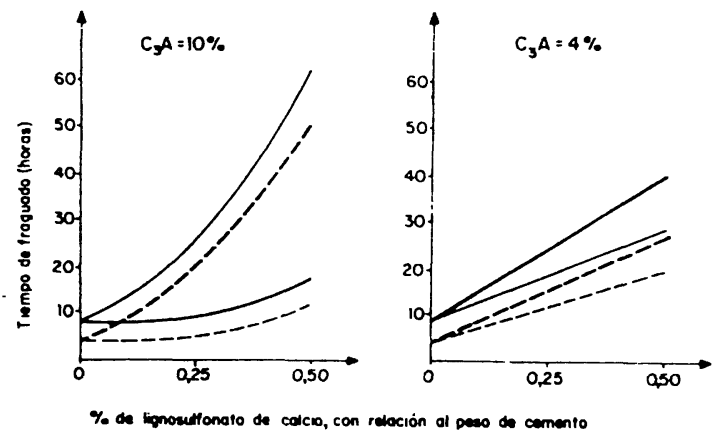

\section{Frogodo}

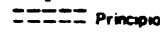

= Fined

Adrain

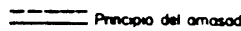

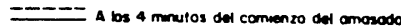

Fig. 2.- Adición de lignosulfonato de calcio. Tiempo de fraguado (pasta de cemento).Influencia del contenido de $C_{f} A$ en el cemento y del modo de adición del aditivo. (Venuat, $M$.: Adjuvants et traitements de mortiers et bétons. Paris, 1971; pág. 159).

relación agua/cemento dada o reduciendo la cantidad de agua de amasado para una consistencia o trabajabilidad determinada o produciendo ambos efectos simultáneamente,

* los tiempos de fraguado y endurecimiento,

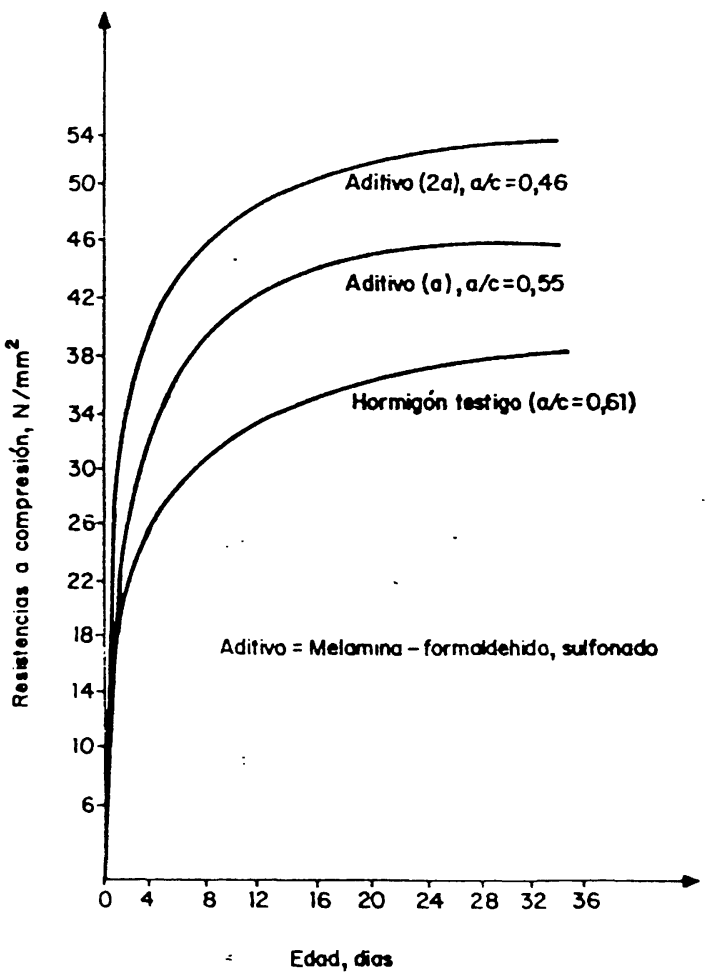

Fïg. 3.- Hormigón: Evolución de las resistencias mecánicas, a compresión, en función del tiempo. Influencia de la cantidad de aditivo. (Massazza, F. e Testolin, M.: I cemento, 1980, 2, 129).

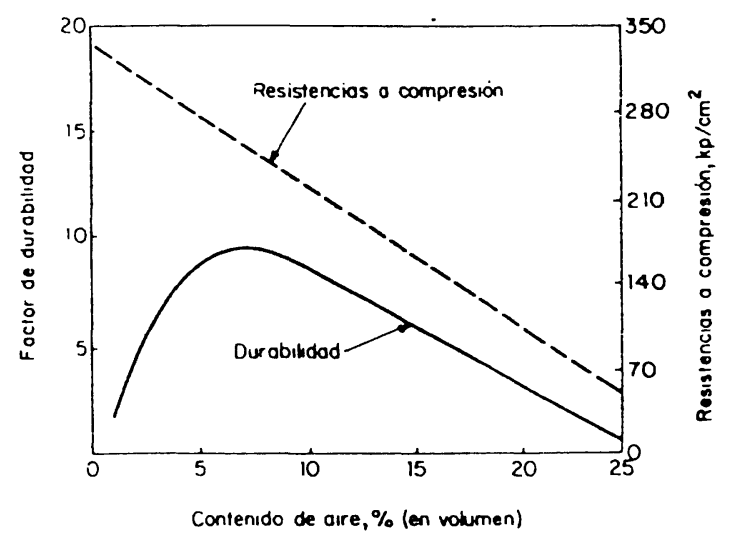

Fig. 4.- Hormigón: Durabilidad. Influencia del contenido de aire. Monders, S. and Young, J.F.: Concrete. London, 1981, pág. 179).

* las resistencias mecánicas,

* la durabilidad química, física o mecánica,

* el contenido de aire o de otros gases,

* otras propiedades.

(Figuras 1, 2, 3 y 4) 
Los mencionados productos (aditivos) empleados correctamente, y añadidos en pequeña cantidad, pueden modificar alguna $o$ algunas de las propiedades del hormigón en sentido favorable, pero -además- dichos productos pueden producir otras modificaciones no deseables que deben ser conocidas, fundamentalmente, por los usuarios de estos productos (fabricantes de hormigón).

De aquí, la conveniencia de utilizar los aditivos sólo después de conocer sus efectos, de un modo especial en un hormigón o mortero definido (hormigón o mortero de referencia), para lo cual es necesario realizar los estudios correspondientes y, a ser posible, efectuar otros estudios empleando materiales análogos a los que se vayan a utilizar en la obra, así como las condiciones de uso establecidas, ya que el empleo de un aditivo adecuado puede influir en una o más propiedades del hormigón afectando, en determinados casos, a ciertas características importantes de un modo favorable o adverso.

Por otra parte, se debe tener en consideración que la dosis de los aditivos, junto con las condiciones de empleo, la naturaleza y características de los cementos y de los áridos, influyen en la acción de un determinado aditivo, de tal modo que pueden actuar en un sentido (positivo) o en otro (negativo), por lo que se impone la necesidad de controlar rigurosamente la dosificación de los aditivos, con objeto de poder optimizar la relación calidad/precio en la formulación de los hormigones. Ahora bien, se debe tener en cuenta que los aditivos no pueden proporcionar una solución favorable para transformar una mala formulación del hormigón en una formulación correcta, del mismo modo que una mala fabricación del hormigón o una puesta en obra deficiente no se pueden transformar en operaciones adecuadas.

\section{3.- ADITIVOS. DEFINICIÓN Y FUNCIONES}

Según la norma española UNE 83-200-84, los aditivos se definen como:

"Aquellas sustancias o productos que incorporados al hormigón, mortero o pasta antes o durante el amasado y/o durante un amasado suplementario, en una proporción no superior al $5 \%$ del peso de cemento (salvo casos especiales), producen la modificación deseada en dicho hormigón, mortero o pasta -en estado fresco $y / o$ endurecido-de alguna de sus características, de sus propiedades habituales o de su comportamiento",

y el prEN 934-2:1992, que se ha sometido a encuesta recientemente entre los miembros del CEN, como:
"Producto incorporado en el momento del amasado del hormigón, en una dosis inferior o igual al $5 \%$ en masa del contenido en cemento del hormigón, con el fin de modificar las propiedades de la mezcla en estado fresco o endurecido".

La prenorma ENV 206, mencionada anteriormente, define a los aditivos en el punto 3.14, del apartado 3 , como:

"Producto que se añade en pequeña proporción con relación a la masa de cemento, antes o durante el amasado, o durante una operación suplementaria al amasado, produciendo las modificaciones deseadas de las caracteristicas normales del hormigón".

En el punto 5.8, del apartado 5, de dicha ENV 206 se fija la cantidad total de aditivo, que se reseña en el apartado 6.3.3..

Desde este punto de vista, y teniendo en cuenta estas definiciones, cabe señalar que otros materiales tales como cenizas volantes, puzolanas, escorias, humo de sílice que pueden ser componentes del cemento (de acuerdo con la normativa actual) y/o del hormigón, así como aquellos que actúan como refuerzos (fibras metálicas, de polipropileno, etc.) no se clasifican como aditivos.

De acuerdo con estas definiciones -que en esencia son coincidentes- un aditivo se caracteriza por producir la modificación de alguna o algunas de las propiedades características del hormigón; bien entendido, de una característica y solamente una, como se menciona en la norma UNE. Esta propiedad se define como FUNCIÓN PRINCIPAL.

Por otra parte, los aditivos pueden modificar accesoriamente alguna, 0 algunas, de las propiedades 0 características del hormigón, independientemente de la que define la función principal. Esta propiedad se define como FUNCIÓN o FUNCIONES SECUNDARIAS.

Además, los aditivos pueden producir modificaciones inevitables de ciertas propiedades o características del hormigón, que no se requieren como funciones secundarias; por ejemplo, débil descenso de las resistencias mecánicas, retraso del tiempo de fraguado, etc. Esta propiedad se define como EFECTO o EFECTOS SECUNDARIOS.

Así, un aditivo puede actuar como reductor de agua del hormigón y, además, puede producir un adelanto del fraguado y una ligera disminución de las resistencias mecánicas. Si este aditivo se va a utilizar como reductor de agua, se debe tener en cuenta lo siguiente: 
* Función principal: ser reductor de agua.

* Función secundaria: ser acelerador de fraguado.

* Efecto secundario: producir débil descenso de las resistencias.

Por consiguiente, este aditivo se debe comercializar -en estos casos- como aditivo reductor de agua y no como acelerador de fraguado.

Como es lógico, definida la función principal de los aditivos, ésta ha permitido su clasificación según que:

a.- modifiquen:

* la reología de los hormigones,

* los tiempos de fraguado y de endurecimiento,

* el contenido de aire o de otros gases;

b.- mejoren:

* la durabilidad química, física o mecánica;

c.- modifiquen o mejoren:

* otras propiedades (p. ej. el color, el bombeo, la proyección, etc. del hormigón).

\section{4.- ADITIVOS. CONTROL DE CALIDAD}

El control de los aditivos se hace cada vez más necesario por diversas razones, pero fundamentalmente con objeto de evitar la utilización de aquellos productos cuyas propiedades y efectos sobre los hormigones no se conozcan con la suficiente garantía.

Para conseguir aditivos de calidad es necesario que el fabricante, en primer lugar, realice su propio control de un modo permanente, de tal modo que pueda garantizar una calidad y una eficacia constante de los productos que suministra a lo largo del tiempo. Para ello, debe establecer los controles correspondientes en las materias primas que utilice, desechando aquellas que no sean aptas (o reciclándolas), así como de los diversos procesos de producción y de los productos

elaborados, de tal modo que éstos respondan a la calidad fijada previamente.

Pero, además, si se quiere garantizar la calidad de dichos productos y, muy especialmente, el porvenir de esta industria, se necesita la existencia de centros o laboratorios especializados con personal adecuado que tenga amplio conocimiento de la fisicoquímica de los cementos y de los restantes componentes del hormigón, así como de su elaboración, transporte y puesta en obra.
Para poder determinar las características de los distintos tipos o familias de aditivos es necesario disponer de los procedimientos operatorios adecuados, así como de las especificaciones, limitaciones, campo de aplicación, etc. de los aditivos, que deben estar recogidos en las normas correspondientes.

\section{5.- NORMAS ESPAÑOLAS UNE}

Conscientes los fabricantes y usuarios españoles de aditivos de proceder a la normalización de definiciones relacionadas con dichos productos y de procedimientos operatorios para determinar las características de los aditivos, de los morteros, hormigones y pastas fabricados con dichos aditivos, así como a la normalización de los diferentes tipos o familias de aditivos, en donde se recojan las especificaciones correspondientes tanto de los aditivos, como de las pastas, morteros y hormigones (con y sin aditivos) y las condiciones de empleo para conseguir los efectos deseados -ya que la incorporación de los mismos sin control, ni estudios apropiados, habia dado lugar a un determinado número de accidentes, fáciles de evitar si se hubiesen usado con el conocimiento debido- se creó en el seno del Comité Técnico de Normalización (CTN) $n^{\circ} 83$ "Hormigón" del AENOR un Subcomité (SC-2 "Aditivos"), al que se le encomendó la misión de preparar la normativa correspondiente.

Como consecuencia de los trabajos desarrollados, se han editado las normas españolas UNE siguientes:

1.- UNE 83-200-84.- Aditivos para hormigones, morteros y pastas. Clasificación y definiciones.

2.- UNE 83-205-85.- Aditivos para hormigones, morteros y pastas. Determinación del residuo seco, $\mathrm{a}(105 \pm 3)^{\circ} \mathrm{C}$, de los aditivos líquidos.

3.- UNE 83-206-85.- Aditivos para hormigones, morteros y pastas.Determinación de la pérdida de masa, a $(105 \pm 3)^{\circ} \mathrm{C}$, de los aditivos sólidos.

4.- UNE 83-207-85.- Aditivos para hormigones, morteros y pastas.

Determinación de la pérdida por calcinación, a $(1050 \pm 25)^{\circ} \mathrm{C}$.

5.- UNE 83-208-85.- Aditivos para hormigones, morteros y pastas. Determinación del residuo insoluble en agua destilada.

6.- UNE 83-209-86.- Aditivos para hormigones, morteros y pastas. Determinación del contenido de agua no combinada. 
7.- UNE 83-210-88 (EXPERIMENTAL).- Aditivos para hormigones, morteros y pastas. Determinación del contenido de halogenuros totales.

8.- UNE 83-211-87 (EXPERIMENTAL).- Aditivos para hormigones, morteros y pastas. Determinación del contenido de compuestos de azufre.

9.- UNE 83-212-89 (EXPERIMENTAL).- Aditivos para hormigones, morteros y pastas.

Determinación del contenido de reductores (poder reductor).

10.- UNE 83-225-86.- Aditivos para hormigones, morteros y pastas. Determinación del peso específico de los aditivos líquidos.

11.- UNE 83-226-86.- Aditivos para hormigones, morteros y pastas. Determinación de la densidad aparente de los aditivos sólidos.

12.- UNE 83-227-86.- Aditivos para hormigones, morteros y pastas. Determinación del $\mathrm{pH}$.

13.- UNE 83-240-86.- Aditivos para hormigones, morteros y pastas. Obtención del espectro infrarrojo.

14.- UNE 83-254-87 (EXPERIMENTAL).- Aditivos para hormigones, morteros y pastas. Toma de muestras.

15.- UNE 83-255-89 (EXPERIMENTAL).- Aditivos para hormigones, morteros y pastas. Materiales testigos y de referencia. Definiciones.

16.- UNE 83-256-87 (EXPERIMENTAL).- Aditivos para hormigones, morteros y pastas. Materiales testigos. Agua de amasado y curado.

17.- UNE 83-257-87 (EXPERIMENTAL).- Aditivos para hormigones, morteros y pastas. Materiales testigos. Áridos.

18.- UNE 83-258-88 (EXPERIMENTAL).- Aditivos para hormigones, morteros y pastas. Morteros. Determinación de la consistencia por medio de la mesa de sacudidas.

19.- UNE 83-259-87 (EXPERIMENTAL).- Aditivos para hormigones, morteros y pastas. Morteros. Determinación del contenido de aire ocluido.

20.- UNE 83-260-89 (EXPERIMENTAL).- Aditivos para hormigones, morteros y pastas.
Determinación del tiempo de fraguado en morteros.

21.- UNE 83-275-89 (EXPERIMENTAL).- Aditivos para hormigones, morteros y pastas. Etiquetado.

22.- UNE 83-281-90 (EXPERIMENTAL).- Aditivos para hormigones, morteros y pastas. Reductores de agua/fluidificantes.

23.- UNE 83-282-90 (EXPERIMENTAL).- Aditivos para hormigones, morteros y pastas. Superfluidificantes/reductores de agua de alta actividad.

24.- UNE 83-283-90 (EXPERIMENTAL).- Aditivos para hormigones, morteros y pastas. Aceleradores de fraguado.

25.- UNE 83-284-90 (EXPERIMENTAL).- Aditivos para hormigones, morteros y pastas. Retardadores de fraguado.

26.- UNE 83-299-92 (EXPERIMENTAL).- Aditivos para hormigones, morteros y pastas. Productos de curado para morteros y hormigones.

Determinación de la pérdida de agua por evaporación.

\section{6.- PROYECTOS DE NORMAS EUROPEAS}

El Grupo de Trabajo n 3 (GT-3) “Admixtures" del Comité Técnico $n^{\circ} 104$ del Comité Europeo de Normalización (CEN/TC 104) "Béton Performance. Production, mise en place et critéres de conformité" ha preparado diversos documentos relacionados con los métodos de ensayo y otros documentos en donde se recogen o recogerán las definiciones correspondientes, las especificaciones y criterios de conformidad, así como la toma de muestras de los aditivos.

\section{1.- Métodos de ensayo}

\subsection{1.- prEN en fase de encuesta realizada}

La descripción de los métodos de ensayo normalizados figura en los proyectos de norma europea que se citan a continuación y que se sometieron a encuesta en el año 1991, excepto el prEN 480-1 que ha sido en abril de 1993:

prEN 480-1:1993.- Admixtures for concrete, mortars and grouts - Test methods - Part 1: Reference concrete and reference mortar for testing. 
prEN 480-2:1991.- Admixtures for concrete, mortar and grout -Test methods - Part 2: Determination of setting time.

prEN 480-3:1991.- Admixtures for concrete, mortar and grout - Test methods - Part 3: Determination of schrinkage and expansion.

prEN 480-4:1991.- Admixtures for concrete, mortar and grout - Test methods - Part 4: Determination of bleeding.

prEN 480-5:1991.- Admixtures for concrete, mortar and grout - Test methods - Part 5: Determination of capillary absorption.

prEN 480-6:1991.- Admixtures for concrete, mortar and grout - Test methods - Part 6: Infrared analysis.

prEN 480-7:1991.- Admixtures for concrete, mortar and grout - Test methods - Part 7: Determination of density of liquid admixtures.

prEN 480-8:1991.- Admixtures for concrete, mortar and grout - Test methods - Part 8: Determination of the conventional dry material content.

prEN 480.9:1991.- Admixtures for concrete, mortar and grout - Test methods - Part 9: Determination of the $\mathrm{pH}$ value.

prEN 480-10:1991.-Admixtures for concrete, mortar and grout - Test methods - Part 10: Determination of the chloride content.

\subsection{2 prEN en fase de encuesta}

prEN 480-11:1994.- Admixtures for concrete, mortar and grout - Determination of air void characteristics in hardened concrete.

prEN 480-12:1994.- Admixtures for concrete, mortar and grout - Determination of the alkali content of admixtures.

\section{2.- Aditivos para el hormigón, morteros y pastas}

Dentro de este grupo de normas se encuentra una serie constituida por las siete partes que se relacionan a continuación:

Parte 1.- Definiciones y exigencias generales para todos los tipos de aditivos.

Parte 2.- Aditivos para hormigón (es el prEN 934-2).

Parte 3.- Aditivos para morteros.
Parte 4.- Aditivos para pastas de inyección

Parte 5.- Aditivos para hormigón proyectado.

Parte 6.- Control de calidad.

Parte 7.- Atestación de conformidad.

Las partes 1 y 3 a 7 están actualmente en fase de preparación por el GT-3 del CEN/TC-104 y la parte 2 -a la que se le ha dado preferencia por haberlo considerado de mayor interés, dada la aplicación de los aditivos en la elaboración del hormigón- se ha sometido a encuesta el pasado mes de mayo.

\section{3.- Aditivos para el hormigón. prEN 934-2:1992}

Como se ha señalado en el punto anterior el proyecto prEN 934-2, titulado "Admixtures for concrete, mortars and grouts - Part 2: Concrete admixtures -Definitions, specifications and conformity criteria", se ha sometido a encuesta recientemente (mayo 1993).

\subsection{1.- Objeto y campo de aplicación}

El prEN 934-2 tiene por objeto definir los aditivos que se utilizan en la fabricación de hormigones, fijar sus especificaciones y los procedimientos operatorios que se deben aplicar para determinar las características especificadas, así como los criterios de conformidad.

Este prEN se aplica a los aditivos para hormigones en masa (no armados), para hormigones armados y pretensados tanto para los fabricados en obra, como para los prefabricados y fabricados en una central (listos para su empleo).

Nota. - En este prEN se define a los aditivos tal como se ha indicado en el apartado 2.

\subsection{2.- Tipos de aditivos}

Los tipos de aditivos incluidos en esta norma, de acuerdo con su función principal en cada caso, así como su definición, son los siguientes:

* Plastificantes/reductores de agua.- Aditivos que, sin modificar la trabajabilidad, permiten reducir la cantidad de agua de una mezcla dada de un hormigón, o que, sin modificar el contenido de agua, aumentan la trabajabilidad, o que producen los dos efectos a la vez.

Nota. - Estos aditivos se denominan en la norma UNE 83-281 fluidificantes/reductores de agua.

* Superplastificantes/reductores de agua de alta actividad-Aditivos que, sin modificar la trabajabilidad, permiten reducir fuertemente el 
contenido de agua de una mezcla dada de hormigón, o que, sin modificar el contenido de agua, aumentan considerablemente la trabajabilidad de la mezcla, 0 que producen los dos efectos a la vez.

Nota. - Estos aditivos se denominan en la norma UNE 83-282 superfluidificantes/reductores de agua de alta actividad.

* Retenedores de agua.- Aditivos que reducen la pérdida de agua disminuyendo la exudación.

* Inclusores de aire. - Aditivos que permiten, durante el amasado del hormigón, la incorporación de una cantidad controlada de finas burbujas de aire uniformemente repartidas, que subsisten después del endurecimiento.

* Aceleradores de fraguado. - Aditivos que disminuyen el tiempo de transición de la mezcla para pasar del estado plástico al estado rígido.

* Aceleradores de endurecimiento. - Aditivos que aceleran el desarrollo de las resistencias iniciales del hormigón, con o sin modificación de la duración del fraguado.

* Retardadores de fraguado. - Aditivos que aumentan el tiempo de transición de la mezcla para pasar del estado plástico al estado rígido.

* Hidrófugos de masa. - Aditivos que disminuyen la absorción capilar del hormigón endurecido.

\subsection{3.- Dosificación}

Dentro de este apartado se incluyen estos tres conceptos:

* Dosificación de conformidad. Es la dosificación, expresada en \% de la masa del cemento, indicada por el fabricante del aditivo. Debe satisfacer las exigencias de este prEN sobre las especificaciones correspondientes a las prestaciones.

La dosificación de conformidad se debe situar en el entorno de dosificación recomendada.

* Entorno de dosificación recomendada. - E1 fabricante debe recomendar los límites de esta dosificación para el producto considerado, con la reserva de tener en cuenta los resultados obtenidos en los ensayos de control llevados a cabo con los componentes que se han de utilizar en la obra.

La dosificación recomendada no debe sobrepasar los límites que figuran en la prenorma ENV 206:90, es decir: “... $50 \mathrm{~g}$ por $\mathrm{kg}$ de cemento contenido en la mezcla, siendo conveniente que esta cantidad no sea inferior a $2 \mathrm{~g}$ por $\mathrm{kg}$ de cemento.

Cantidades menores de aditivos sólamente se autorizan si se dispersan en una cierta proporción del agua de amasado.

Los aditivos líquidos, cuyas cantidades sean superiores a 3 litros $/ \mathrm{m}^{3}$ de hormigón se deben tener en cuenta en el cálculo de la relación agua/cemento".

* Dosificación máxima recomendada.- Es el límite superior del entorno de dosificaciones, que se utiliza para los ensayos de compatibilidad.

\subsection{4.- Identificación, compatibilidad, prestaciones. Definiciones}

* Identificación: Es un procedimiento que permite caracterizar los aditivos y controlar su uniformidad lote por lote, verificando su similitud química y física, tal como se indica en este prEN.

* Compatibilidad: Es la aptitud para ser utilizado un aditivo con diferentes cementos.

* Prestaciones: Es la aptitud de un aditivo para ser eficaz en la utilización prevista, sin producir efectos perjudiciales.

\subsection{5.- Especificaciones}

Las especificaciones de los diferentes tipos de aditivos relacionadas con su identificación y conformidad de compatibilidad figuran en la tabla 4 y las de conformidad en las tablas 5.1 a 5.8. (Págs. 55 a 59).

Las especificaciones de las prestaciones para cada tipo de aditivos, que tienen por objeto limitar determinados efectos secundarios de los aditivos y que se refieren a las funciones principales, se incluyen en las tablas 5.1 a 5.8 .

a) Identificación de los aditivos.- Todos los tipos de aditivos se identifican a partir de las especificaciones de las siguientes características:

* homogeneidad (segregación), color,

* compuesto efectivo (IR),

* densidad real,

* extracto seco convencional,

* valor del pH, 
que figuran en la tabla 4, titulada "Conformity criteria and required information".

b) Compatibilidad de los aditivos.- Como ensayo de compatibilidad para todos los diferentes tipos de aditivos (para ser utilizado un aditivo con diferentes cementos) únicamente se considera el tiempo de fraguado (tabla 4).

c) Prestaciones. Especificaciones.- Las características especificadas para los diferentes tipos de aditivos son las siguientes:

c.1) Características especificadas para todos los tipos de aditivos:

* Contenido en cloruros.

* Contenido en álcalis ( $\mathrm{Na}_{2} \mathrm{O}$, equivalente).

* Contenido en aire en el hormigón fresco.

* Comportamiento a la corrosión.

Nota.- Se señala que los aditivos no deben favorecer la corrosión del acero embebido en el hormigón.

* Resistencia a compresión (Nota.- Se debe incluir en la tabla 5.1)

c.2) Características especificadas para determinados tipos de aditivos:

* Reducción de agua, para los aditivos.-Plastificantes/ reductores de agua. Superplastificantes/reductores de agua de alta actividad.

* Incremento de consistencia para los aditivos.Plastificantes/reductores de agua. Nota.- Se debe incluir en la tabla 5.1). Superplastificantes/ reductores de agua de alta actividad.

* Mantenimiento de la trabajabilidad (consistencia) para los aditivos.- Superplastificantes/reductores de agua de alta actividad.

\section{* Tiempo de fraguado para los aditivos.} Aceleradores de fraguado. Retardadores de fraguado.

* Exudación para los aditivos. - Retenedores de agua.

*Absorción capilar para los aditivos.-Hidrófugos.

c.3) Las especificaciones que deben cumplir cada uno de los diferentes tipos de aditivos figuran en las tablas 5.1 a 5.8, ambas inclusive, tituladas "Conformity requirements". d) Métodos de ensayo

d.1) Identificación de los aditivos.- Los métodos de ensayo de las características para identificar a un aditivo figuran en la tabla 1, titulada "Test methods for identification requirements".

d.2) Compatibilidad de los aditivos.- Los métodos de ensayo relativos a las características de compatibilidad de los diferentes tipos de aditivos se encuentran en la tabla 2, titulada "Test methods for compatibility requirements".

La compatibilidad de los aditivos del hormigón se valora por los ensayos realizados con los morteros definidos en el prEN 480, parte 1, y preparados como se especifica en dicho prEN, utilizando la dosificación máxima recomendada del aditivo con cuatro cementos, como los que se especifican en prEN 480-1 (párrafo 3.1.2).

Nota. - Los cementos especificados en prEN 480-1, para los ensayos de compatibilidad, son los siguientes: CEM I, CEM II, CEM III, CEM IV, definidos en la prenorma ENV 197-1, que se corresponden con los cementos tipo I, II, III y IV de la norma UNE 80.301.

Los cementos elegidos deben ser de tipo diferente y/o de procedenicia distinta.

d.3) Prestaciones.- Los métodos de ensayo para determinar las especificaciones de las prestaciones de un aditivo se incluyen en tabla 3, titulada "Test methods for performance requirements".

Las prestaciones de los aditivos para el hormigón se determinan a partir de los ensayos realizados con los morteros y hormigones de referencia, que se describen en el prEN 480-1, obtenidos de acuerdo con las condiciones especificadas en dicho prEN, utilizando un cemento CEM I, de la clase de resistencia 42,5, según ENV 197-1 (cemento tipo I/42,5, según UNE 80.301).

e) Criterios de conformidad e informaciones.- Las especificaciones de conformidad para cada característica de las prestaciones correspondientes a cada uno de los ocho tipos de aditivos, definidos en este prEN 934-2, se encuentran en las tablas 5.1 a 5.8 , ambas inclusive.

Cada tipo de aditivo debe cumplir con las especificaciones de las prestaciones correspondientes a las características que figuran en las tablas 5.1 a 5.8 ya mencionadas. 
Table 1

Test methods for identification requirements

\begin{tabular}{|c|l|l|}
\hline No. & \multicolumn{1}{|c|}{ REQUIREMENT } & \multicolumn{1}{|c|}{ TEST METHOD } \\
\hline 1 & Homogenity and Colour & visual \\
\hline 2 & Effective Component (Infrared Analysis) & prEN 480 Part 6 \\
\hline 3 & Relative Density & prEN 480 Part 7 \\
\hline 4 & Conventional dry material content & prEN 480 Part 8 \\
\hline 5 & pH value & prEN 480 Part 9 \\
\hline
\end{tabular}

Table 2

Test methods for compatibility requirements

\begin{tabular}{|c|l|l|}
\hline No. & \multicolumn{1}{|c|}{ REQUIREMENT } & \multicolumn{1}{c|}{ TEST METHOD } \\
\hline 6 & Setting time & prEN 480 Part 2 \\
\hline
\end{tabular}

Table 3

Test methods for performance requirements

\begin{tabular}{|c|l|l|}
\hline No. & \multicolumn{1}{|c|}{ REQUIREMENT } & \multicolumn{1}{c|}{ TEST METHOD } \\
\hline 7 & Chloride content & prEN 480 Part 10 \\
\hline 8 & Alkali content & has to be elaborated \\
\hline 9 & Air content in fresh concrete & ISO 4848 \\
\hline 10 & Compressive strength & ISO 4012 \\
\hline 11 & Water reduction & $\begin{array}{l}\text { ISO 4109 (slump) - ISO 9812 } \\
\text { (flow) }\end{array}$ \\
\hline 12 & Increase in consistency & $\begin{array}{l}\text { ISO 4109 (slump) - ISO 9812 } \\
\text { (flow) }\end{array}$ \\
\hline 13 & Consistency retention & $\begin{array}{l}\text { ISO 4109 (slump) - ISO 9812 } \\
\text { (flow) }\end{array}$ \\
\hline 14 & Air content in hardened concrete (air void spacing) & prEN 480 Part 11 \\
\hline 15 & Setting time & prEN 480 Part 2 \\
\hline 16 & Capillary absorption & prEN 480 Part 5 \\
\hline 17 & Bleeding & prEN 480 Part 6 \\
\hline
\end{tabular}


Table 4

Conformity criteria and required information

Identification and Compatibility

Definition clause 4.2.1. to 4.2.8. for all admistures

\begin{tabular}{|c|c|}
\hline IDENTIFICATION & REQUIREMENTS \\
\hline Segregation, Colour & $\begin{array}{ll}\text { Segregation: } & \text { shall not be more than the manufacturers stated value } \\
\text { Colour: } & \text { shall be visually uniform and similar to the colour of the } \\
& \text { reference sample }\end{array}$ \\
\hline Effective component (IR) & $\begin{array}{l}\text { Infrared spectra shall have similar characteristic peaks with the } \\
\text { corresponding relative absorption of the reference sample }\end{array}$ \\
\hline Relative density & $\begin{array}{l}\text { Manufactures stated value } \pm 0,03 \text { if stated value is above } 1,10 \text { and } \pm \\
0,02 \text { if value is equal or below } 1,10\end{array}$ \\
\hline Conventional dry material & $\begin{array}{l}0,95 \mathrm{~T} \leq \mathrm{X} \% \leq 1,05 \mathrm{~T} \\
\mathrm{~T}: \text { manufacturers value; } \mathrm{X} \text { : test value }\end{array}$ \\
\hline pH-value & $\begin{array}{l}\text { Manufacturers stated value } \pm 1 \text { or within the range stated by } \\
\text { manufacturer }\end{array}$ \\
\hline
\end{tabular}

\begin{tabular}{|l|l|}
\hline \multicolumn{1}{|c|}{ COMPATIBILITY } & \\
\hline $\begin{array}{l}\text { Setting time at MRD } \\
\text { (maximum recommended dosage) }\end{array}$ & $\begin{array}{l}\text { No requirements, but to report results with } 4 \text { cements as specified in } \\
\text { prEN 480 Part 1 section 3.1.2. }\end{array}$ \\
\hline
\end{tabular}

Note (1): not final

Table 5.1

Conformity requirements

Type: Water reducing/plasticizing admixture

Definition clause 4.2.1.

\begin{tabular}{|l|l|}
\hline \multicolumn{1}{|c|}{ REQUIREMENT } & \multicolumn{1}{c|}{ PERFORMANCE } \\
\hline $\begin{array}{l}\text { Chloride-content } \\
(\mathrm{Cl})\end{array}$ & $\begin{array}{l}\text { Not above the maximun content specified by the manufacturer or to } \\
\text { conform with the manufacturers claim that it is less than } 0,10 \% \text { by mass }\end{array}$ \\
\hline $\begin{array}{l}\text { Alkali-content } \\
\left(\mathrm{Na}_{2} \mathrm{O} \text { equivalent }\right)\end{array}$ & Not more than the manufacturers stated maximum value \\
\hline Air content in fresh concrete & $\begin{array}{l}\text { Not more than } 2 \% \text { above reference mix unless specifically stated } \\
\text { otherwise by the manufacturer }\end{array}$ \\
\hline Water reduction & $\geq 5 \%$ at equal consistency, as determined by slump or flow \\
\hline Setting time & N/R \\
\hline
\end{tabular}


Table 5.2

Conformity requirements

Type: High range water-reduction/superplasticizing

Definition clause 4.2.2.

\begin{tabular}{|l|l|}
\hline \multicolumn{1}{|c|}{ REQUIREMENT } & \multicolumn{1}{c|}{ PERFORMANCE } \\
\hline $\begin{array}{l}\text { Chloride-content } \\
(\mathrm{Cl})\end{array}$ & $\begin{array}{l}\text { Not above the maximun content specified by the manufacturer or to } \\
\text { conform with the manufacturers claim that it is less than } 0,10 \% \text { by mass }\end{array}$ \\
\hline $\begin{array}{l}\text { Alkali-content } \\
\left(\mathrm{Na}_{2} \mathrm{O} \text { equivalent }\right)\end{array}$ & Not more than the manufacturers stated maximum value \\
\hline Air content in fresh concrete & $\begin{array}{l}\text { Not more than } 2 \% \text { above reference mix unless specifically stated } \\
\text { otherwise by the manufacturer }\end{array}$ \\
\hline Compressive strength & $\begin{array}{l}\text { High range water-reduction } \\
1 \text { day at least } 140 \% ; 28 \text { days at least } 115 \% \text { of reference mix } \\
\text { Superplasticiser: at least } 90 \% \text { of reference mix at } 28 \text { days }\end{array}$ \\
\hline Water reduction & $\geq 12 \%$ determined by slump or flow \\
\hline Increase consistency & $\begin{array}{l}\text { Increase in flow } \geq 160 \mathrm{~mm} \text { from initial flow } 380 \pm 20 \mathrm{~mm} \\
\text { Increase in slump } \geq 120 \mathrm{~mm} \text { from initial slump } 70 \pm 10 \mathrm{~mm}\end{array}$ \\
\hline Workability consistency & $\begin{array}{l}30 \text { min after the addition, the consistency shall not fall to the value of the } \\
\text { initial consistency of the reference mix }\end{array}$ \\
\hline
\end{tabular}

Table 5.3

Conformity requirements

Type: water retaining

Definition clause 4.2.3.

\begin{tabular}{|l|l|}
\hline \multicolumn{1}{|c|}{ REQUIREMENT } & \multicolumn{1}{c|}{ PERFORMANCE } \\
\hline $\begin{array}{l}\text { Chloride-content } \\
(\mathrm{Cl})\end{array}$ & $\begin{array}{l}\text { Not above the maximun content specified by the manufacturer or to } \\
\text { conform with the manufacturers claim that it is less than } 0,10 \% \text { by mass }\end{array}$ \\
\hline $\begin{array}{l}\text { Alkali-content } \\
\left(\mathrm{Na}_{2} \mathrm{O} \text { equivalent }\right)\end{array}$ & Not more than the manufacturers stated maximum value \\
\hline Air content in fresh concrete & $\begin{array}{l}\text { Not more than } 2 \% \text { above reference mix unless specifically stated } \\
\text { otherwise by the manufacturer }\end{array}$ \\
\hline Compressive strength & $\geq 80 \%$ of reference mix at 28 days \\
\hline Bleeding & A reduction of at least $50 \%$ compared with special reference mix \\
\hline
\end{tabular}


Table 5.4

Conformity requirements

Type: Air entraining

Definition clause 4.2.4.

\begin{tabular}{|l|l|}
\hline \multicolumn{1}{|c|}{ REQUIREMENT } & \multicolumn{1}{c|}{ PERFORMANCE } \\
\hline $\begin{array}{l}\text { Chloride-content } \\
(\mathrm{Cl})\end{array}$ & $\begin{array}{l}\text { Not above the maximun content specified by the manufacturer or to } \\
\text { conform with the manufacturers claim that it is less than } 0,10 \% \text { by mass }\end{array}$ \\
\hline $\begin{array}{l}\text { Alkali-content } \\
\left(\mathrm{Na}_{2} \mathrm{O} \text { equivalent }\right)\end{array}$ & Not more than the manufacturers stated maximum value \\
\hline Air content in fresh concrete & $5,5 \pm 0,5 \%$ \\
\hline $\begin{array}{l}\text { Air content in hardened concrete } \\
\text { (air void spacing) }\end{array}$ & Spacing factor $\leq 0,200 \mathrm{~mm}$ \\
\hline Compressive strength & $\geq 75 \%$ of reference mix at 28 days \\
\hline
\end{tabular}

Table 5.5

Conformity requirements

Type: set accelerating

Definition clause 4.2.5.

\begin{tabular}{|l|l|}
\hline \multicolumn{1}{|c|}{ REQUIREMENT } & \multicolumn{1}{c|}{ PERFORMANCE } \\
\hline $\begin{array}{l}\text { Chloride-content } \\
(\mathrm{Cl})\end{array}$ & $\begin{array}{l}\text { Not above the maximun content specified by the manufacturer or to } \\
\text { conform with the manufacturers claim that it is less than } 0,10 \% \text { by mass }\end{array}$ \\
\hline $\begin{array}{l}\text { Alkali-content } \\
\left(\mathrm{Na}_{2} \mathrm{O} \text { equivalent }\right)\end{array}$ & Not more than the manufacturers stated maximum value \\
\hline Air content in fresh concrete & $\begin{array}{l}\text { Not more than } 2 \% \text { above reference mix unless stated otherwise by the } \\
\text { manufacturer }\end{array}$ \\
\hline Compressive strength & $\begin{array}{l}28 \text { days strength of test mix } \geq 80 \% \text { of reference mix } \\
90 \text { days strength of test mix } \geq \text { the compressive strength of test mix at } 28 \\
\text { days }\end{array}$ \\
\hline Setting time & $\begin{array}{l}\text { Initial set } \geq 30 \text { min for test mix, at } 20^{\circ} \mathrm{C} \text { reduction } \geq 40 \% \text { at } 5^{\circ} \mathrm{C} \\
\text { compared with reference mix }\end{array}$ \\
\hline
\end{tabular}


Table 5.6

Conformity requirements

Type: hardening accelerating

Definition clause 4.2.6.

\begin{tabular}{|l|l|}
\hline \multicolumn{1}{|c|}{ REQUIREMENT } & \multicolumn{1}{c|}{ PERFORMANCE } \\
\hline $\begin{array}{l}\text { Chloride-content } \\
(\mathrm{Cl})\end{array}$ & $\begin{array}{l}\text { Not above the maximun content specified by the manufacturer or to } \\
\text { conform with the manufacturers claim that it is less than } 0,10 \% \text { by mass }\end{array}$ \\
\hline $\begin{array}{l}\text { Alkali-content } \\
\left(\mathrm{Na}_{2} \mathrm{O} \text { equivalent }\right)\end{array}$ & Not more than the manufacturers stated maximum value \\
\hline Air content in fresh concrete & $\begin{array}{l}\text { Not more than } 2 \% \text { above reference mix unless specifically stated } \\
\text { otherwise by the manufacturer }\end{array}$ \\
\hline Compressive strength & $\begin{array}{l}\text { at } 20^{\circ} \mathrm{C} \geq 120 \% \text { of reference mix at } 24 \mathrm{~h} 90 \% \text { or reference mix at } 28 \\
\text { days } \\
\text { at } 5^{\circ} \mathrm{C} \geq 130 \% \text { of reference mix at } 48 \mathrm{~h}\end{array}$ \\
\hline
\end{tabular}

Table 5.7

Conformity requirements

Type: set retarding

Definition clause 4.2.7.

\begin{tabular}{|l|l|}
\hline \multicolumn{1}{|c|}{ REQUIREMENT } & \multicolumn{1}{c|}{ PERFORMANCE } \\
\hline $\begin{array}{l}\text { Chloride-content } \\
(\mathrm{Cl})\end{array}$ & $\begin{array}{l}\text { Not above the maximun content specified by the manufacturer or to } \\
\text { conform with the manufacturers claim that it is less than } 0,10 \% \text { by mass }\end{array}$ \\
\hline $\begin{array}{l}\text { Alkali-content } \\
\left(\mathrm{Na}_{2} \mathrm{O} \text { equivalent }\right)\end{array}$ & Not more than the manufacturers stated maximum value \\
\hline Air content in fresh concrete & $\begin{array}{l}\text { Not more than } 2 \% \text { above reference mix unless specifically stated } \\
\text { otherwise by the manufacturer }\end{array}$ \\
\hline Compressive strength & $\begin{array}{l}\geq 80 \% \text { of reference mix at } 7 \text { days } \\
\geq 90 \% \text { of reference mix at } 28 \text { days }\end{array}$ \\
\hline Setting time & $\begin{array}{l}\text { Initial setting time } \geq 90 \text { min greater than reference mix } \\
\text { Final setting time } \leq 360 \text { min greater than reference mix }\end{array}$ \\
\hline
\end{tabular}


Table 5.8

Conformity requirements

Type: water repelling

Definition clause 4.2.8.

\begin{tabular}{|l|l|}
\hline \multicolumn{1}{|c|}{ REQUIREMENT } & \multicolumn{1}{c|}{ PERFORMANCE } \\
\hline $\begin{array}{l}\text { Chloride-content } \\
(\mathrm{Cl})\end{array}$ & $\begin{array}{l}\text { Not above the maximun content specified by the manufacturer or to } \\
\text { conform with the manufacturers claim that it is less than } 0,10 \% \text { by mass }\end{array}$ \\
\hline $\begin{array}{l}\text { Alkali-content } \\
\left(\mathrm{Na}_{2} \mathrm{O} \text { equivalent }\right)\end{array}$ & Not more than the manufacturers stated maximum value \\
\hline Air content in fresh concrete & $\begin{array}{l}\text { Not more than } 2 \% \text { above reference mix unless specifically stated } \\
\text { otherwise by the manufacturer }\end{array}$ \\
\hline Compressive strength & $\geq 85 \%$ of reference mix at 28 days \\
\hline Capillary absorption: either & $\begin{array}{l}\text { At } 7 \text { days } \leq 50 \% \text { of reference mix for test duration, } 1 \text { and } 7 \text { days } \\
\text { At } 90 \text { days } \leq 60 \% \text { of reference mix for test duration, } 1,7 \text { and } 28 \text { days for } \\
\text { equal consistency } \\
\text { St } 7 \text { and } 90 \text { days } \leq 80 \% \text { of reference mix for test duration, } 1,7 \text { and } 28 \\
\text { days for equal water to cement ratio }\end{array}$ \\
\hline
\end{tabular}

\section{f) Observaciones}

f. 1) En la tabla 5.1 (plastificantes/reductores de agua) se deben indicar las resistencias a compresión, del mismo modo que se hace en la tabla 5.2

(superplastificantes/reductores de agua de alta actividad).

f.2) El contenido de aire en el hormigón fresco con aditivo debe ser $(5,5 \pm 0,5) \%$ y el factor de espaciado de las burbujas de aire en el hormigón endurecido de la misma mezcla no debe ser superior a 0,200 $\mathrm{mm}$. En el caso de que la dosificación de conformidad no se pueda fijar, la dosificación se debe ajustar de tal manera que se obtenga el contenido de aire requerido (según prEN 934-2).

f.3) El tiempo de fraguado se determina en morteros. El método de ensayo se encuentra descrito en el prEN 480, parte 2 .

\section{CONCLUSIONES}

Primera: Los aditivos han tenido gran influencia en el desarrollo de la industria del hormigón, así como en la aparición de nuevas técnicas de preparación del mismo y de puesta en obra.

Segunda: El control de los aditivos se hace cada vez más necesario por diversas razones, pero

-fundamentalmente- para evitar el empleo de aquellos productos cuyas propiedades y efectos sobre los hormigones no se conozcan con la suficiente garantía.

Tercera: Para garantizar la calidad de los aditivos es necesario disponer de la normativa correspondiente sobre las características y especificaciones correspondientes, así como sobre los procedimientos operatorios para determinarlas.

Cuarta: Todos los aditivos deben cumplir con los resultados de los ensa-yos correspondientes a las características de identificación y compatibilidad especificadas.

Quinta: Cada aditivo debe cumplir con los resultados de los ensayos de prestaciones de las características de conformidad especificadas. 


\section{BIBLIOGRAFÍA CONSULTADA}

* La normativa que figura en el texto.

* Adjuvants (Rapport final).- Commission 11 A RILEM.- Mat. et Const.; Essais et Recherches,1975,48,451-472.

* Chemical Admixtures for Concrete. Reported by ACI Committee 212. ACI 212.3R-89.

* Guide d'emploi des adjuvants du béton. AFREM “Adjuvants”. Travaux,1987,juillet-août,14-21.

* Guide to chemical admixtures for concrete. Concrete Society Technical Report 18. London,1980.

* Guide for Use of Admixtures in Concrete. Reported by ACI Committee 212. ACI 212.2R-81 (Rev.1986).

* FERNANDEZ CANOVAS, M. Utilización de aditivos en el hormigón de carreteras. II Symp. sobre aditivos para el hormigón. ANFAH. Barcelona. 1985

* GARCIA MESEgUER, A. Control de calidad y garantía de calidad. Seminario S.1 del X curso CEMCO. Madrid,1985.

* GASPAR TÉBAR, D. Aditivos. Clasificación y normativa. I Symp. sobre aditivos para el hormigón. ANFAH. Madrid,1983.

* GASPAR TÉBAR, D. Aditivos. Estado actual de la normativa en España. II Symp. sobre aditivos para el hormigón. ANFAH. Barcelona, 1985. Cemento-Hormigón, 1987, 640, 237-276.

* GASPAR TÉBAR, D. Aditivos para hormigón, morteros y pastas. Calidad y Normativa. Cemento-Hormigón,1987, 644, 694-722.

* GASPAR TÉBAR, D. Aditivos para el hormigón. La normalización en España y la futura normativa europea. III Symp. sobre aditivos para el hormigón. ANFAH. Madrid, 1988.

* GASPAR TÉBAR, D. Aditivos para el hormigón. ANCOP. Madrid, 1989.

* GALARON, A. Utilización de los aditivos en el hormigonado de grandes presas. II Symp. sobre aditivos para el hormigón. ANFAH. Barcelona, 1985.

* PAILLERE, A. M., BEN BASSAT, M. and AKMAN, S. "Guide for use of admixtures in concrete". Mat. and Struct., 1992, 25, 49-56.

* PAILLERE, A. M. Sellos de calidad en los aditivos de hormigón (en Francia). I Symp. sobre aditivos para el hormigón, ANFAH, Madrid,1983.

* PAILLERE, A. M. Les adjuvants comme moyen d'adaptation aux resources et conditions locales. Curso postgrado E.T.S.I.C. Barcelona, 1986.

* RIXOM, M. R. Chemical Admixtures for Concrete. E.\& F.N. Spon Ltd. London, 1978.

* RIXOM, M. R. Aditivos para los hormigones (traducido por E. Herrero). Edit.Técnic. Asoc., S.A. Barcelona, 1983.

* VENUAT, M. Adjuvants et Traitements des Mortiers et Bétons. París, 1971. 